\title{
Quantum well saturable absorber mirror with electrical control of modulation depth
}

\author{
Liu, Xiaomin; Rafailov, E.U.; Livshits, D.; Turchinovich, Dmitry
}

Published in:

2010 Conference on Lasers and Electro-Optics (CLEO) and Quantum Electronics and Laser Science Conference (QELS)

Publication date:

2010

Document Version

Publisher's PDF, also known as Version of record

Link back to DTU Orbit

Citation (APA):

Liu, X., Rafailov, E. U., Livshits, D., \& Turchinovich, D. (2010). Quantum well saturable absorber mirror with electrical control of modulation depth. In 2010 Conference on Lasers and Electro-Optics (CLEO) and Quantum Electronics and Laser Science Conference (QELS) (pp. 1-2). IEEE. http://www.cleoconference.org/

\section{General rights}

Copyright and moral rights for the publications made accessible in the public portal are retained by the authors and/or other copyright owners and it is a condition of accessing publications that users recognise and abide by the legal requirements associated with these rights.

- Users may download and print one copy of any publication from the public portal for the purpose of private study or research.

- You may not further distribute the material or use it for any profit-making activity or commercial gain

- You may freely distribute the URL identifying the publication in the public portal 


\title{
Quantum Well Saturable Absorber Mirror with Electrical Control of Modulation Depth
}

\author{
Xiaomin Liu ${ }^{1}$, Edik U. Rafailov ${ }^{2}$, Daniil Livshits ${ }^{3}$, \\ and Dmitry Turchinovich ${ }^{1 *}$ \\ ${ }^{1}$ DTU Fotonik - Department of Photonics Engineering, Technical University of Denmark, \\ DK-2800 Kgs. Lyngby, Denmark \\ ${ }^{2}$ Photonics and Nanoscience, University of Dundee, Dundee DD1 4HN, UK \\ ${ }^{3}$ Innolume GmbH, Konrad-Adenauer-Allee 11, Dortmund, 44263, Germany \\ *dmtu@fotonik.dtu.dk
}

\begin{abstract}
A saturable absorber mirror comprizing InGaAs/GaAs quantum wells incorporated into a $p-i-n$ structure is demonstrated. Its modulation depth can be reduced from $4.25 \%$ to $1.63 \%$ by applying reverse bias voltage in the range $0-1 \mathrm{~V}$.

(C) 2009 Optical Society of America

OCIS codes: (250.5590) Quantum-well, -wire and -dot devices; (140.7090) Ultrafast lasers; (140.4050) Mode-locked lasers
\end{abstract}

\section{Introduction}

Semiconductor saturable absorber mirrors (SESAMs) are used for modelocking of ultrafast solid-state and fiber lasers [1, 2]. Modulation depth - the value of saturable loss of SESAM, is an important parameter governing selfstarting and sustainability of modelocking. In this work we demonstrate a quantum well-based SESAM for $\mathrm{Yb}$ wavelength, incorporated into a $p-i-n$ structure. The SESAM modulation depth can be changed in the range $4.25 \%$ to $1.63 \%$ by applying a reverse bias voltage in the range $0-1 \mathrm{~V}$. We believe that this effect may find its application in ultrafast laser control and stabilization both because its strength and simplicity of implementation in the lasers.

\section{SESAM design and experimental results}

Our SESAM was grown on a GaAs substrate, followed by $500 \mathrm{~nm}$ GaAs buffer and AlGaAs/GaAs Bragg reflector. The absorber InGaAs/GaAs quantum well $(\mathrm{QW})$ region followed the Bragg reflector, and was capped by $\mathrm{AlGaAs} / \mathrm{GaAs}$ cap layer. The QWs width was $8 \mathrm{~nm}$. The GaAs buffer and the Bragg reflector were $\mathrm{n}$-doped, the QW region was undoped, and the cap layer was p-doped, thus resulting in a $p-i-n$ structure. The schematic of the SESAM is shown in Fig. 1(a). The Bragg reflector provided high reflectivity in the range 1020-1120 nm. The peak absorption of the QWs was around $1056 \mathrm{~nm}$, and $3 \mathrm{~dB}$ reduction of peak absorption was at the wavelengths of $1038 \mathrm{~nm}$ and $1080 \mathrm{~nm}$. We used narrow stripe electrodes separated by approximately $300 \mu \mathrm{m}$ on the cap layer, which provided free
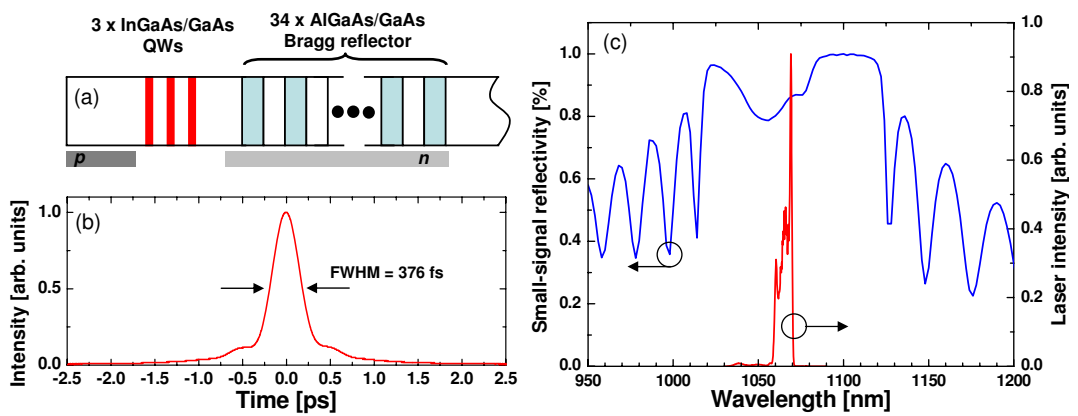

Fig. 1: (a) Schematic of an electrically controlled SESAM. (b) Autocorrelation of the laser pulse used in the experiments. (c) Small-signal reflectivity of the SESAM at zero bias, and spectrum of the laser.

optical access to SESAM. Similar electrically controlled SESAMs were already used in modelocking of picosecond solid-state lasers. For example, in the work [3], pulse shortening from 17.4 ps to 6.4 ps, and spectral shift from 1280 $\mathrm{nm}$ to $1282 \mathrm{~nm}$ were observed with application of the reverse bias.

However, the laser is a complex system, where SESAM action is in interplay with gain saturation, gain curvature, and other wavelength- and power-dependent loss factors. Therefore, a more direct experiment is needed for character- 


\section{CThI2.pdf}

ization of SESAM action alone. In our work, we directly measured the reflected power, spectrum, and autocorrelation of femtosecond $\mathrm{Yb}$-fiber laser pulses upon reflection from the SESAM, as a function of pumping fluence and reverse bias voltage. The details of the laser used in these experiments can be found in [4]. The laser pulses had autocorrelation full width at half maximum (FWHM) of $376 \mathrm{fs}$ (See. Fig. 1(b)), corresponding to the pulse duration of about $280 \mathrm{fs}$. The spectrum of the laser, centered around $1065 \mathrm{~nm}$, is shown in Fig. 1(c) along with the small-signal reflectivity of the SESAM at zero bias.

In our experiments we did not observe neither the noticeable spectral modification, nor the significant pulse shortening upon the reflection from the SESAM as compared to the reference Au mirror in all range of pumping fluences 6-47 $\mu \mathrm{J} / \mathrm{cm}^{2}$ and reverse bias voltages $0-2 \mathrm{~V}$, used in our measurements.
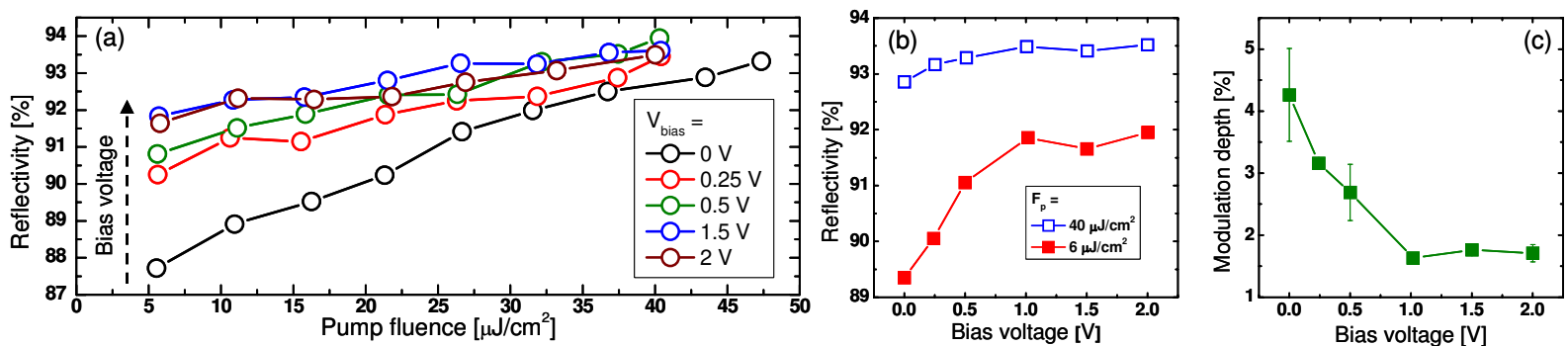

Fig. 2: (a) Reflectivity of SESAM as a function of pump fluence, at different values of reverse bias voltage in the range 0-2 V. (b) Reflectivity of the SESAM at pump fluence of $6 \mu \mathrm{J} / \mathrm{cm}^{2}$ and $40 \mu \mathrm{J} / \mathrm{cm}^{2}$, as a function of reverse bias voltage. (c) Modulation depth of the SESAM as a function of reverse bias voltage.

However, we discovered a strong change in the saturable loss of the SESAM when the reverse bias voltage was applied. The reflectivity dependence on the pump fluence for several values of reverse bias voltage is shown in Fig. 2(a). In Fig. 2(b) the SESAM reflectivities at pump fluences of $6 \mu \mathrm{J} / \mathrm{cm}^{2}$ and $40 \mu \mathrm{J} / \mathrm{cm}^{2}$ as a function of reverse bias voltage are shown. We define the modulation depth as the difference in the reflectivities at these two pump fluences. In Fig. 2(c) the SESAM modulation depth as a function of reverse bias voltage is shown. It decreases from $4.25 \%$ down to $1.63 \%$ by applying a reverse bias voltage in the range $0-1 \mathrm{~V}$, and saturates at higher bias values.

The SESAM saturable loss for low-fluence signals can be strongly reduced by applying reverse bias, whereas for the high-fluence signals this change is much smaller (see Fig. 2(b)). Therefore, it is the overall reduction of absorption strength of the QWs with applied reverse bias voltage, that should play a crucial role in the observed effect.

At the critical reverse bias voltage of $1 \mathrm{~V}$, the electric field experienced by the QWs in addition to the built-in field of $p-i-n$ junction is $66 \mathrm{kV} / \mathrm{cm}$ for our structure. Such a strong electric field will result in a strong additional quantumconfined Stark effect on the QWs [5], leading to the redshift of the QW absorption line (by confinement potential tilt) and reduction of the QW absorption strength (by separating the electron and hole wavefunctions). Since the laser spectrum is placed at the longer-wavelength side of the QW absorption maximum at zero bias voltage, more applied reverse bias could have lead to an increase of low-fluence laser absorption by "moving" the QW absorption line into the laser spectrum, which was not the case here. Therefore, it is probably the dominating contribution of wavefunction separation, that causes the observed effect of reduced absorption of low-fluence signals.

In conclusion, we demonstrated a significant (by a factor of 2.6) reduction of modulation depth of the quantum well SESAM for $\mathrm{Yb}$ wavelength from $4.25 \%$ to $1.63 \%$, by applying reverse bias voltage in the range $0-1 \mathrm{~V}$. Applied reverse bias did not affect neither the pulse shape nor the spectrum of 280-fs long pulses at $1065 \mathrm{~nm}$, used in our experiments, within the accuracy of our measurements. We believe that an observed effect of electric control of SESAM modulation depth may find its application in ultrafast laser control and stabilization, in particular in active stabilization of fiber lasers against Q-switched modelocking, provided the structure with higher initial modulation depth is realized.

\section{References}

1. U. Keller, "Recent developments in compact ultrafast lasers," Nature (London) 424, 831-838 (2003).

2. O. Shtyrina, M. Fedoruk, S. Turitsyn, R. Herda, and O. Okhotnikov, "Evolution and stability of pulse regimes in SESAM-mode-locked femtosecond fiber lasers," J. Opt. Soc. Am. B 26, 346-352 (2009).

3. S.A. Zolotovskaya, K.G. Wilcox, A. Abdolvand, D.A.Livshits, and E.U. Rafailov, "Electronically controlled pulse duration passively modelocked Cr : Forsterite laser," IEEE Photon. Technol. Lett. 21, 1124-1126 (2009).

4. D. Turchinovich, X. Liu, and J. Lægsgaard, "Monolithic all-PM femtosecond Yb-fiber laser stabilized with a narrow-band fiber Bragg grating and pulse-compressed in a hollow-core photonic crystal fiber," Opt. Express 16, 14004-140014 (2008).

5. D.A.B. Miller, D.S. Chemla, T.C. Damen, A.C. Gossard, W. Wiegmann, T.H. Wood, and C.A.Burrus, "Band-edge electroabsorption in quantum well structures - the quantum-confined Stark-effect,", Phys. Rev. Lett. 53, 2173-2176 (1984). 